\title{
Tuning the performance of bulk metallic glasses by milling artificial \\ holes
}

\author{
Feng Gong a, Shun Hua Chen ${ }^{\mathrm{b}}$, Jia Qi Ran ${ }^{\text {a }}$, Zhi Yang a, Jiang Ma ${ }^{\mathrm{a}, *}$ \\ ${ }^{a}$ Key Laboratory of Advanced Manufacturing Technology for Mold and Die, College \\ of Mechatronics and Control Engineering, Shenzhen University, Shenzhen 518060, \\ PR China \\ ${ }^{b}$ Advanced Manufacturing Technology Research Centre, Department of Industrial \\ and Systems Engineering, The Hong Kong Polytechnic University, \\ Hung Hom, Hong Kong
}

\begin{abstract}
The mechanical performance of materials is greatly affected and could be tuned by artificial defects, especially for amorphous alloys. In present work, specially designed holes are created for bulk metallic glass and apparent mechanical performance improvement is obtained when compared with the intact ones. The fracture characterization discovers that the inner wall of the artificial hole has a blocking effect to shear bands (SBs), leading to an apparent enhancement of mechanical property. Our results demonstrate that the blocking effect of SBs induced by the designing artificial hole may provide some new sights on the plastic deformation mechanism of metallic glasses rather than the improved plasticity itself.
\end{abstract}

Keywords: bulk metallic glasses; artificial holes; shear bands

*Author to whom correspondence should be addressed. Email: majiang@szu.edu.cn 


\section{Introduction}

Excellent mechanical performance such as high strength combined with good plasticity is earnestly pursued by people during the seeking of superior materials for engineering applications.[1] As for crystalline alloys, this goal could often be achieved through the reduction of grain size, unfortunately, the incompatibility between plasticity and strength constraints the mechanical performance of materials.[2] What is more, such incompatibility persists in nanocrystalline metals and alloys, which display highly improved strength but very little plastic deformation,[3] and also in bulk metallic glasses (BMGs) with completely disordered atomic structures. The unique properties of BMGs, such as high strength, high specific strength, large elastic strain limit, and excellent wear and corrosion resistances along with other remarkable engineering properties, have made these materials of significant interest for science and industry.[4-18] However, the lack of plasticity makes them prone to catastrophic failure in load-bearing conditions and greatly restricts their wide-spread applications. In order to improve the mechanical performance of BMGs, it is widely accepted that efforts should be made from the following aspects:[19] designing BMGs by modulating their elastic modulus;[20, 21] introducing nano-scale structural heterogeneity;[22] minor alloying some elements with low $\mu / B$ (or, equivalently, high $v$ ) as constituents to induce randomly distributed free volume;[23] tuning the casting conditions;[24] composition design from the structural perspective;[25] designing BMG composites with nanocrystals or 
quasicrystals,[8] ductile dendrites,[26-28] fibers,[29] particles,[30, 31] phase separation,[32] and crystalline layers;[33] forming porous BMGs;[34, 35] and surface treatment [36, 37] or pre-deformation treatment.[38] Previous work also found that plasticity could be achieved by designing artificial defects, such as notches or micro structures.[39-41]

For crystalline materials, the dislocations and slip systems are the key factors controlling the plastic deformation behavior,[39] however, for metallic glasses without lattice dislocations and slip systems, shear banding becomes the significant plastic deformation mechanism[17]. Once yielding starts, the shear bands (SBs) propagate rapidly, resulting in a catastrophic fracture. It is generally accepted that there is a strong connection between the density of shear bands and the plasticity of BMGs[17, 39], therefore, efforts have been made to create enough shear bands in order to improve the property of BMGs. On the other hand, the primary SB which brings the final catastrophic fracture always propagates through the interior of the specimen, if the propagation routine was cut off by artificial defects, shear bands in metallic glasses expand with difficulty when stimulated by designed structures, the entire plasticity should be greatly enhanced. Following such strategies, in the present work, we created a series of through holes in the BMG specimen body, hoping to promote the generation of SBs and make difficulties for them to expand. Compared with the intact specimens, the plasticity of the metallic glass with artificial holes can be clearly increased, however, the yield strength has no apparent decay. Furthermore, the fracture morphologies of different specimens are also characterized and compared 
to find out how the artificial holes affect the mechanical performance of BMGs. The current results demonstrate that the blocking effect of SBs induced by designing artificial through hole may provide some new understanding on the plastic deformation mechanism of metallic glasses rather than the improved plasticity itself.

\section{Experimental details}

Zr-based metallic glass rods with a nominal chemical composition of $\mathrm{Zr}_{35} \mathrm{Ti}_{30} \mathrm{Cu}_{8.25} \mathrm{Be}_{26.75}$, which are prepared by the arc melting and copper mold suck-casting method, were chosen for present research. The final rods have a diameter of $3 \mathrm{~mm}$ and a height of $6 \mathrm{~mm}$. A series of holes with different diameters of $0.9,1.2$, $1.5,1.8,2.1$ and $2.4 \mathrm{~mm}$ were drilled by the milling machine on the right center of the metallic glass rod, resulting different diameter ratio of hole to rod (DR), which were $3: 10,2: 5,1: 2,3: 5,7: 10$ and $4: 5$, respectively. The illustration of the mechanical performance test for the intact and machined specimens are presented in Figure 1(a). To examine if the unexpected crystallization was introduced during the milling, the chips were collected and identified by X-ray diffraction (XRD; Bruker D8 advance) with $\mathrm{Cu} \mathrm{K}_{\alpha}$ radiation, and the result is presented in the inset of Fig. 1(b), it is found that the chips were amorphous, which implies that the inner wall of the hole remains the amorphous nature. The residual stress of the samples after drilling was removed by vacuum annealing at $200{ }^{\circ} \mathrm{C}$ for 1 hour, after that, the final surfaces were polished by the electrochemical method (Struers, LectroPol-5) in order to fully eliminate the influence of the drilling[42]. 


\section{Results and discussion}

The mechanical performance of the BMG specimens was measured on the electromechanical MTS 810 equipment and the stress and strain curves are presented in Fig. 1(b). The aspect ratio of height to diameter was 2:1. To obtain the reliable results, all of the compression tests for the samples under one condition were repeated for five times. It can be seen that the fracture behavior of the as-cast specimen is a typically brittle one, with average fracture strength of $1870 \mathrm{MPa}$ and almost no plasticity. However, the specimens with artificial holes reveal different mechanical performance. In contrast with the intact specimen, the specimens with artificial holes show improvements in plasticity. One can see that the specimens with DRs of 3:10, 2:5 and 1:2 have only little plasticity improvement, which is not obvious. However, as for the specimens with DRs of 3:5, 7:10, the conditions were different, significant improvements on plasticity were achieved, which is not at the cost of the decrease of fracture strength. As it is shown in Fig. 1(b), the strength of the specimens with DRs of 3:5 and 7:10 were 1810 and $1900 \mathrm{MPa}$, respectively. What is more, the plasticity of them was increased from nearly zero of the intact one to $2 \%$ and $4.5 \%$. The artificial holes were not always favorable for the mechanical performance enhancement, when the DR of BMG specimen was increased to $4: 5$, the mechanical performance became bad. The strength was $1732 \mathrm{MPa}$ and nearly no plasticity was observed. Maybe the reason is when the DR value is large, the wall thickness becomes very small and unexpected mechanical failure occurred under compression. The results show that it is an effective approach to tune the mechanical performance of BMGs by drilling 
artificial holes, and this method may provide a new idea to improve the property of those BMG systems without any intrinsic plasticity.

Moreover, the effect of the aspect ratio of height to diameter was also considered. Figure 1(c) presents the stress-strain curves of the specimens with different aspect ratio values of height to diameter, which were $2: 1,4: 3$ and $2: 3$, respectively. These specimens had with a fixed $\mathrm{DR}=0.7$. When the height is small compared with the diameter (e. g. aspect ratio of height to diameter is 2:3), it seems the specimen has a slightly larger plasticity of about $4.6 \%$, only $2 \%$ higher than the specimen with aspect ratio of height to diameter $2: 1$. That is to say, the aspect ratio of height to diameter has limited effect on the mechanical performance of BMG specimens with artificial holes.

To investigate the reason of the improved mechanical performance more clearly, the fracture morphologies of the BMG specimens with artificial holes are characterized by scanning electron microscope (SEM; Hitachi SU-70). The full views of the fracture section of the intact and machined BMG specimens were shown in Fig. 2(a) and (b), and the close-up views of different positions in the full views (highlighted by the dashed zones I, II, III and IV) are presented in Fig. 2(c), (d), (e) and (f), respectively. As for the intact BMG specimen, the morphology around the fracture is shown in Fig. 2(f), one can see the density of the SBs is similar to the corresponding position of the machined BMG specimen (see Fig. 2(d)). The results indicate that the artificial through hole should be responsible for the apparent difference in mechanical performance between two differnt kinds of BMG specimens, and the existence of the through hole in BMG stops the propagation of the generated 
SBs, causing it tougher than the intact one. Another detail that should be noted is the morphology far from the fracture, as presented in Fig. 2(c) and (e) for intact specimen and the machined specimen, respectively. From Fig. 2(c), we can see clear corrugate pattern on the SB, however, similar pattern is not observed in Fig. 2(e), but only a straight SB line can be found. The unique corrugate pattern observed in the machined specimen may be caused by the difficulties that the SBs meet during their propagation.

It can be believed that dense SBs were stimulated around the artificial holes and stopped by the inner wall of the through hole during their propagation. The SEM images of the inner wall (i.e. the dashed region V in Fig. 1(a)) of the machined BMG specimens could be the evidence. Figure 2(g) - (l) present the inner wall SEM images of the machined BMG specimens with different DR values. From Fig. 2(g) to 2(k), the DR values of them are $3: 10,2: 5,1: 2,3: 5$ and 7:10, respectively. An obvious tendency is that the density of generated SBs in the inner wall becomes larger and larger with the increase of DR values. That is to say, the plasticity of these specimens should also follow this tendency, which is just in accordance with the experimental results. However, as for the specimen with a DR value of $4: 5$, the density of SBs in the inner wall (see Fig. 2(1)) decreased instead of growing, which explains the lack of plasticity for this sample. In addition, one can see the density of the SBs in the inner wall of the specimen with plasticity enhancement (e.g. Fig. 2(k)) is much larger than the outside surface of the specimen (e.g. Fig. 2(d) and (f)), if the density of the SBs was estimated by the total length of all SBs per unit area, then the density of SBs in 
the inner wall is approximately 3 times larger than the outside surface. The dense SBs generated in the inner wall of the specimens with artificial through holes play important roles for the enhancement of plasticity.

The interaction between the inner wall of the through hole and the SBs generated during deformation can be illustrated in Fig. 3. For the convenience of observation, the sectional views are used in the illustration. During the process of deformation, SBs were initiated and propagated with the increase of stress. If the specimen is intact, the primary SB would be rapidly formed and spread across the specimen, causing the catastrophic fracture. However, for a specimen with through hole, the initiated SBs are stopped by the inner wall of the hole and the propagation routine of the SBs is also cut off, leading to an enhancement of the mechanical performance.

The mechanical performance improvement of the specimen with through hole may be due to the special stress distributions induced by the artificial defect. To find out the reason, the finite element analysis (FEA) was used. FEA was carried out with the elastic-perfectly plastic model due to no work-hardening for most BMGs,[13] and the results were presented in Fig. 4. From the FEA results, it can be seen that the sample with through hole show evident stress concentrations around the through hole, away from the through hole, the local stresses decrease gradually, forming stress gradients. The closer to the inner wall of the hole, the larger the stress is observed from Fig. 4. Once the local stresses approach to the yield conditions of the BMG, SBs will be easily activated, which coincides well with the locations of the observed SB zones. Hence, the initiation of multiple SBs can be ascribed to the stress concentration 
around the through hole.

\section{Conclusions}

In present work, artificial through holes were introduced to BMGs. The creation of through holes brings apparent mechanical performance improvement to them, which may be due to the easy initiation of SBs around the milled holes, and the consequent blocking effect of holes on the propagation of shear bands, similar to the dislocation mechanism in crystalline materials. Our results may provide some new understanding on the plastic deformation mechanism of metallic glasses rather than the improved plasticity itself.

\section{Acknowledgements}

The authors are grateful for experimental help from discussions with Prof. W. H. Wang. The financial support of the NSF of China (Grant No. 51501116, 51175348, 51205258) and Natural Science Foundation of SZU (Grant No. 836-00008318) are appreciated. 


\section{References}

[1] L. Lu, Y. Shen, X. Chen, L. Qian, K. Lu, Science 304 (2004) 422-426.

[2] J. Schiøtz, K.W. Jacobsen, Science 301 (2003) 1357-1359.

[3] K. Kumar, H. Van Swygenhoven, S. Suresh, Acta Mater. 51 (2003) 5743-5774.

[4] W. Klement, R.H. Willens, P. Duwez, Nature 187 (1960) 869-870.

[5] A.L. Greer, Science 267 (1995) 1947-1953.

[6] W.L. Johnson, MRS bulletin 24 (1999) 42-56.

[7] W.H. Wang, C. Dong, C.H. Shek, Materials Science and Engineering: R: Reports 44 (2004) 45-89.

[8] A. Inoue, Acta Mater. 48 (2000) 279-306.

[9] H.S. Chen, Reports on Progress in Physics 43 (1980) 353.

[10] Z.P. Lu, C.T. Liu, Acta Mater. 50 (2002) 3501-3512.

[11] A. Peker, W.L. Johnson, Appl. Phys. Lett. 63 (1993) 2342-2344.

[12] M.D. Demetriou, M.E. Launey, G. Garrett, J.P. Schramm, D.C. Hofmann, W.L. Johnson, R.O. Ritchie, Nat. Mater. 10 (2011) 123-128.

[13] C. Schuh, T. Hufnagel, U. Ramamurty, Acta Mater. 55 (2007) 4067-4109.

[14] M. Telford, Materials Today 7 (2004) 36-43.

[15] P.F. Guan, M.W. Chen, T. Egami, Phys. Rev. Lett. 104 (2010) 205701.

[16] T. Nieh, J. Wadsworth, C. Liu, T. Ohkubo, Y. Hirotsu, Acta Mater. 49 (2001) 2887-2896.

[17] C.A. Schuh, T.C. Hufnagel, U. Ramamurty, Acta Mater. 55 (2007) 4067-4109.

[18] B.A. Sun, H.B. Yu, W. Jiao, H.Y. Bai, D.Q. Zhao, W.H. Wang, Phys. Rev. Lett. 
$105(2010)$.

[19] J.J. Lewandowski, M. Shazly, A. Shamimi Nouri, Scr. Mater. 54 (2006) 337-341.

[20] Y.H. Liu, G. Wang, R.J. Wang, D.Q. Zhao, M.X. Pan, W.H. Wang, Science 315 (2007) 1385-1388.

[21] J. Schroers, W.L. Johnson, Phys. Rev. Lett. 93 (2004) 255506.

[22] J. Das, M.B. Tang, K.B. Kim, R. Theissmann, F. Baier, W.H. Wang, J. Eckert, Phys. Rev. Lett. 94 (2005) 205501.

[23] L. Chen, Z. Fu, G. Zhang, X. Hao, Q. Jiang, X. Wang, Q. Cao, H. Franz, Y. Liu, H. Xie, Phys. Rev. Lett. 100 (2008) 075501.

[24] K. Yao, F. Ruan, Y. Yang, N. Chen, Appl. Phys. Lett. 88 (2006) 122106.

[25] L. Zhang, Y.-Q. Cheng, A.-J. Cao, J. Xu, E. Ma, Acta Mater. 57 (2009) $1154-1164$

[26] C. Hays, C. Kim, W.L. Johnson, Phys. Rev. Lett. 84 (2000) 2901.

[27] G. He, J. Eckert, W. Löser, L. Schultz, Nat. Mater. 2 (2003) 33-37.

[28] D.C. Hofmann, J.-Y. Suh, A. Wiest, G. Duan, M.-L. Lind, M.D. Demetriou, W.L. Johnson, Nature 451 (2008) 1085-1089.

[29] R. Conner, R. Dandliker, W.L. Johnson, Acta Mater. 46 (1998) 6089-6102.

[30] C. Fan, H. Li, L.J. Kecskes, K. Tao, H. Choo, P. Liaw, C. Liu, Phys. Rev. Lett. 96 (2006) 145506.

[31] Y. Wu, Y. Xiao, G. Chen, C.T. Liu, Z. Lu, Adv. Mater. 22 (2010) 2770-2773.

[32] X. Du, J. Huang, K. Hsieh, Y. Lai, H. Chen, J. Jang, P. Liaw, Appl. Phys. Lett. 91 (2007) 131901. 
[33] Y. Wang, J. Li, A.V. Hamza, T.W. Barbee, Proceedings of the National Academy of Sciences 104 (2007) 11155-11160.

[34] A.H. Brothers, D.C. Dunand, Adv. Mater. 17 (2005) 484-486.

[35] T. Wada, A. Inoue, A.L. Greer, Appl. Phys. Lett. 86 (2005).

[36] Y. Zhang, W. Wang, A. Greer, Nat. Mater. 5 (2006) 857-860.

[37] J. Fan, A. Chen, M. Fu, J. Lu, Scr. Mater. 61 (2009) 608-611.

[38] P. Yu, H. Bai, J. Zhao, C. Jin, W. Wang, Appl. Phys. Lett. 90 (2007) 51906-51906.

[39] J. Zhao, F. Wu, R. Qu, S. Li, Z. Zhang, Acta Mater. 58 (2010) 5420-5432.

[40] R.T. Qu, J.X. Zhao, M. Stoica, J. Eckert, Z.F. Zhang, Materials Science and Engineering: A 534 (2012) 365-373.

[41] R.T. Qu, Q.S. Zhang, Z.F. Zhang, Scr. Mater. 68 (2013) 845-848.

[42] A. L. Greer, Y. Q. Cheng, E. Ma, Materials Science and Engineering: R: 74 (2013) $71-132$. 


\section{Figure Captions}

Fig. 1. (a) Illustration of the intact and through hole BMG samples under compression. (b) Stress-strain curves of the intact and machined BMG specimens with different DR values. The inset presents the XRD pattern of the chips during the milling of holes. (c) Stress-strain curves of the specimens with different aspect ratio values of height to diameter.

Fig. 2. (a) and (b) show the full views of the fracture section of the intact and machined BMG specimens. (c)-(f) present the close-up views of different positions in the full views (highlighted by the dashed zones I, II, III and IV). (g) - (l) present the inner wall SEM images of the machined BMG specimens with different DR values. (h)-(l) share the same scale bar.

Fig. 3. The interaction between the inner wall of the through hole and the SBs generated during deformation.

Fig. 4. Finite element analysis (FEA) results of the Von-Mises stress distribution of the specimen with a through hole. 
(a)
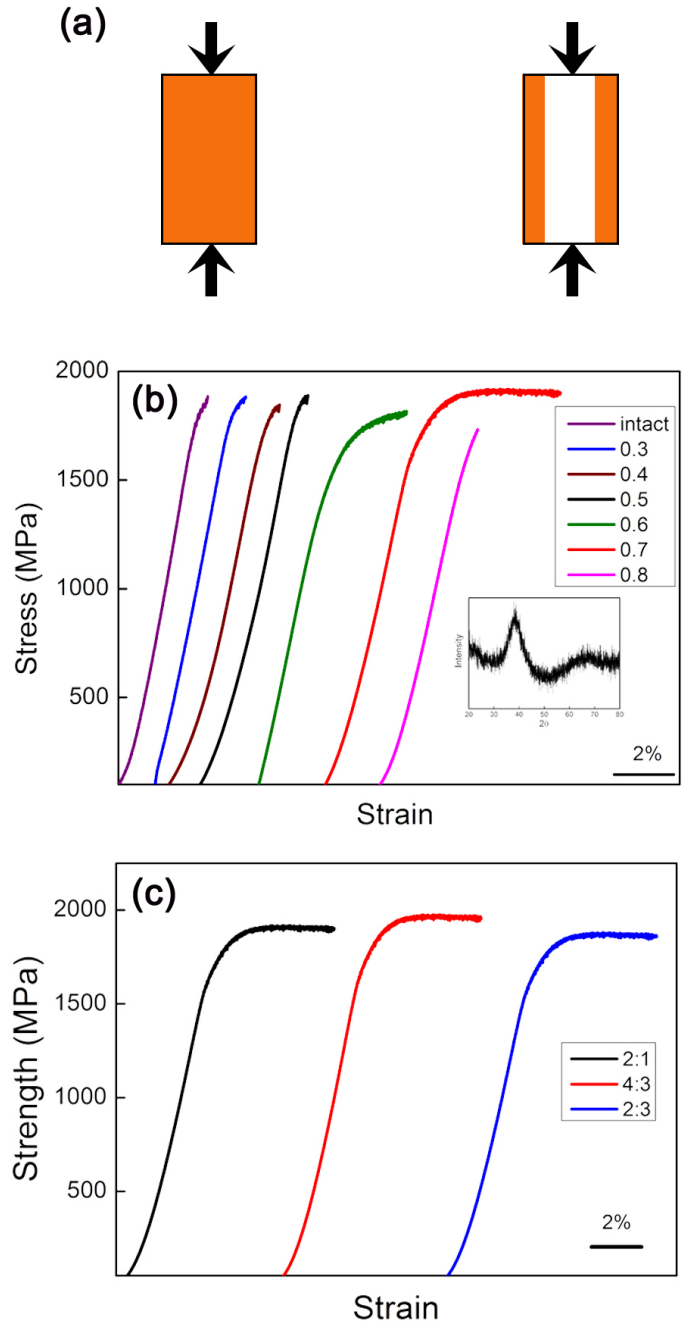

Fig. 1. F. Gong et al 

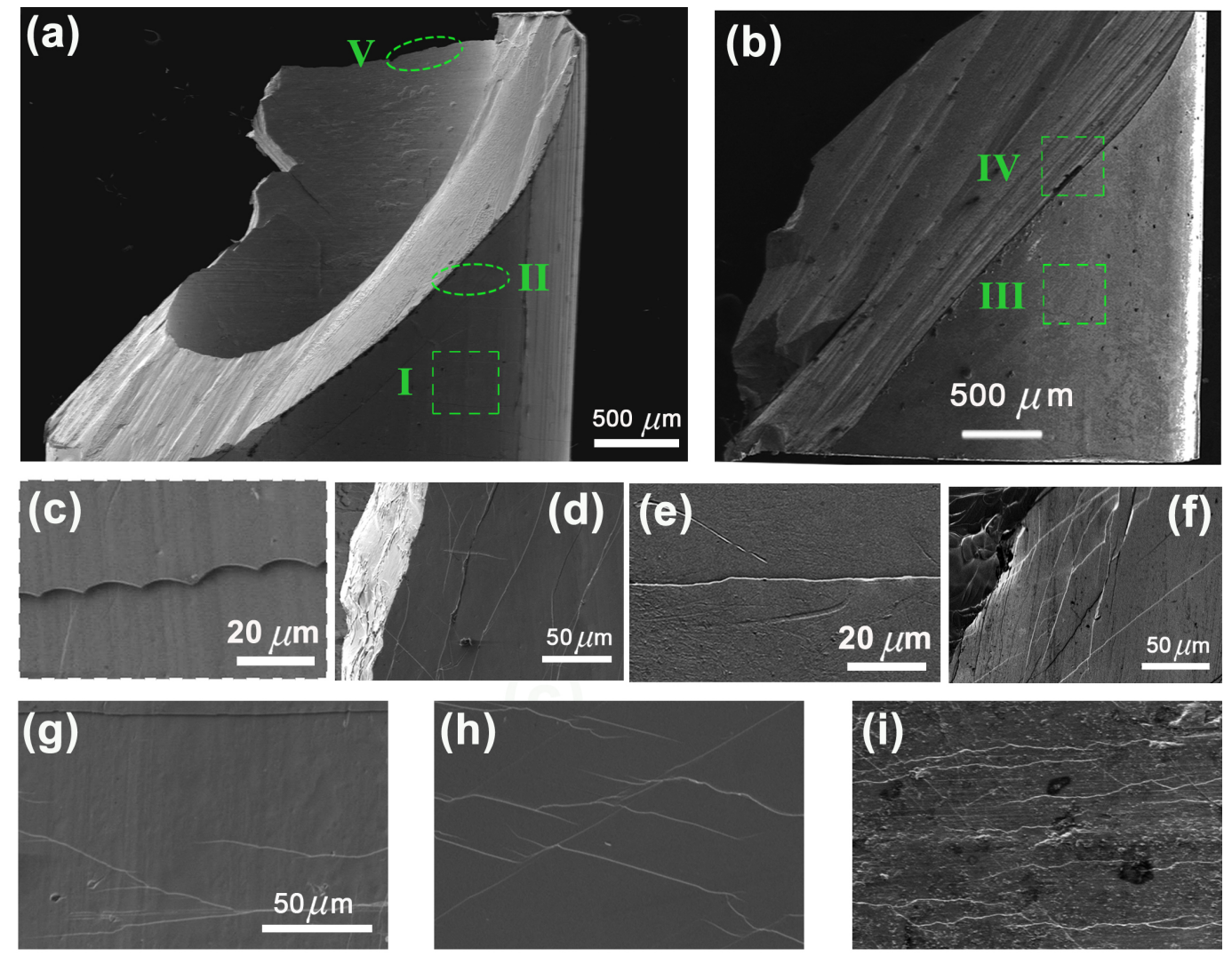

(h)
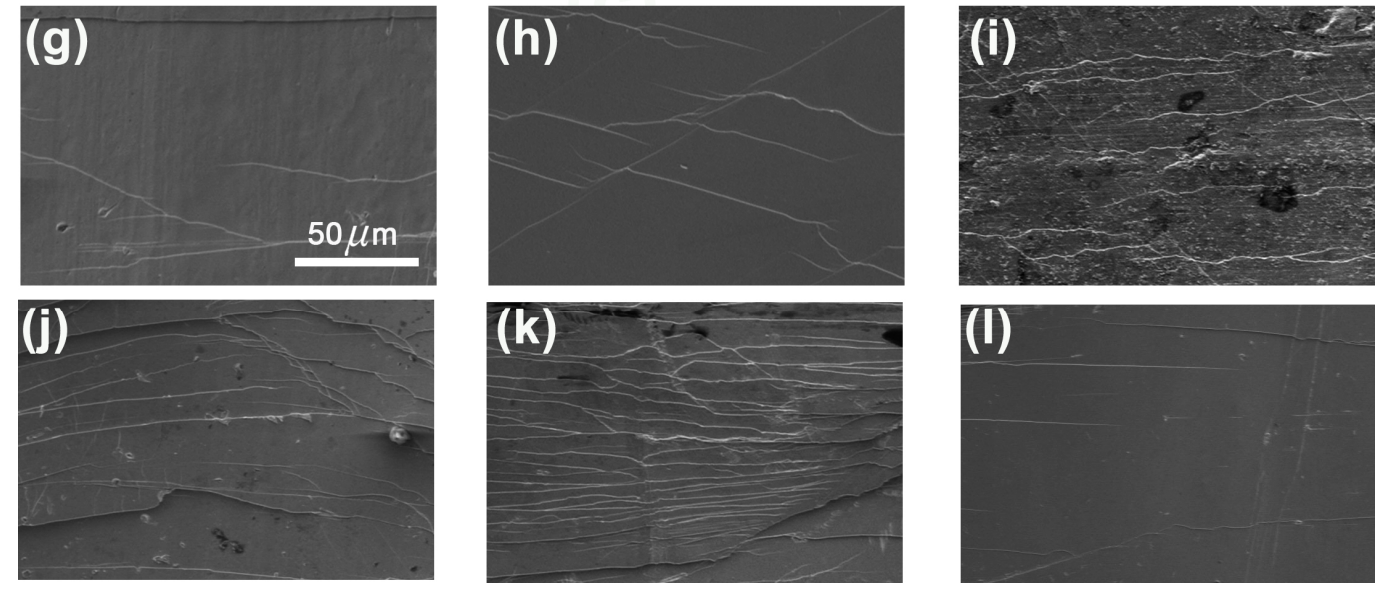

Fig. 2. F. Gong et al 

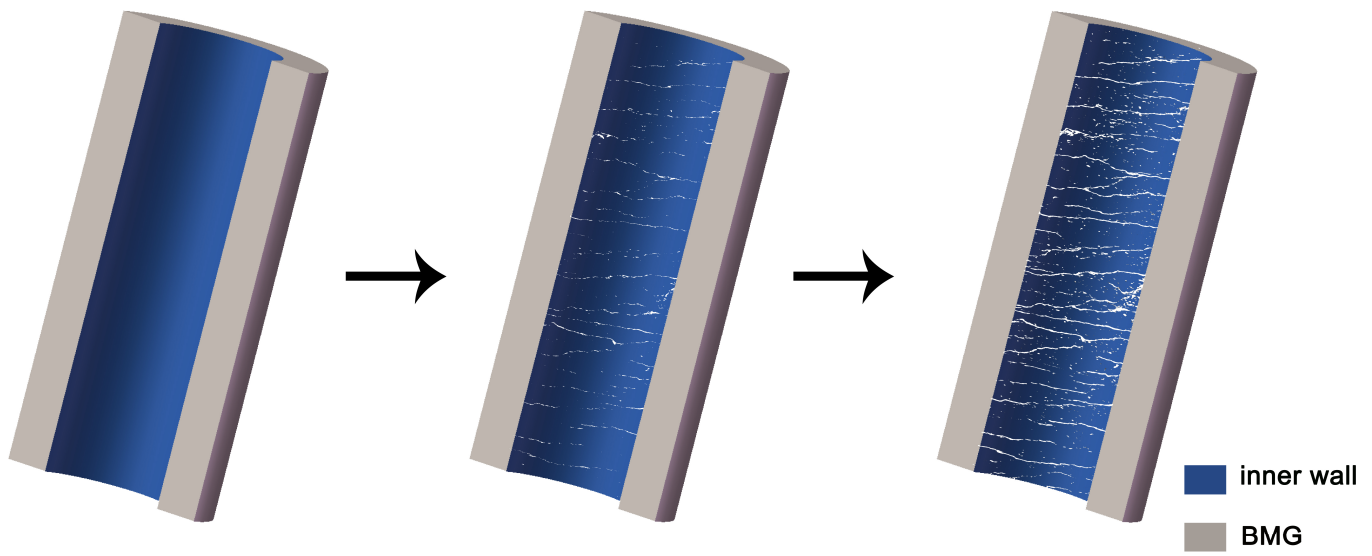

Fig. 3. F. Gong et al 


\begin{tabular}{|c|}
\hline Stress $(\mathrm{Pa})$ \\
\hline$+1.730 \mathrm{e}+09$ \\
\hline $\begin{array}{r}+1.640 \mathrm{e}+09 \\
+1.550 \mathrm{e}+09\end{array}$ \\
\hline $\begin{array}{r}+1.460 \mathrm{e}+09 \\
\end{array}$ \\
\hline $\begin{array}{r}+1.370 \mathrm{e}+09 \\
\end{array}$ \\
\hline$+1.280 \mathrm{e}+09$ \\
\hline $\begin{array}{r}+1.190 \mathrm{e}+09 \\
\end{array}$ \\
\hline $\begin{array}{r}1.100 \mathrm{e}+09 \\
\end{array}$ \\
\hline$-+1.010 \mathrm{e}+09$ \\
\hline $\begin{array}{l}+9.200 \mathrm{e}+08 \\
+8.300 \mathrm{e}+08\end{array}$ \\
\hline $\begin{array}{r}+7.400 \mathrm{e}+08 \\
\end{array}$ \\
\hline$+6.500 \mathrm{e}+08$ \\
\hline
\end{tabular}

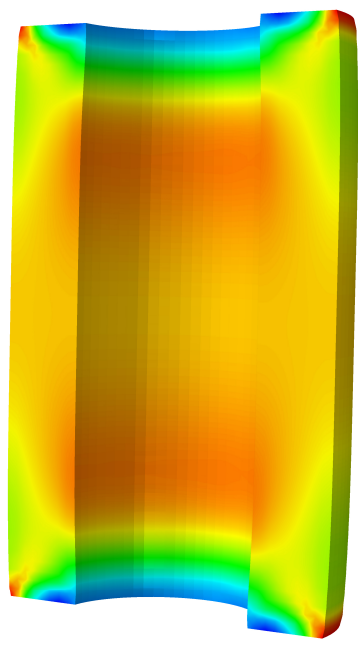

Fig. 4. F. Gong et al 